Manuelle Medizin 2010 · 48:5

DOI 10.1007/s00337-010-0749-z

๑) Springer-Verlag 2010

\author{
J. Meyer zu Tittingdorf \\ Springer Medizin Heidelberg
}

\section{Julius-Springer-Forschungspreis Manuelle Medizin}

\section{Forschung im Sinne des Patienten}

Liebe Leserinnen und Leser,

der Springer-Verlag als wissenschaftlicher Fachverlag versteht sich seit seiner Gründung im Jahre 1842 durch Julius Springer auch als Förderer der Forschung und Wissenschaft.

Gerade in der manuellen Medizin wird der Ruf nach einer stärkeren wissenschaftlichen Ausrichtung des Fachgebiets mit der Integration in die Schulmedizin zunehmend lauter. Dieser Ruf gründet sich u. a. darauf, dass Sie als Manualmediziner in Ihrer täglichen Arbeit begonnen haben, die Befunde zu systematisieren und die Therapie komplexer zu gestalten. Und trotz zufriedener Patienten mit Ihrem ärztlichen Handeln begründet sich dieser Ruf auch darin, dass seitens Dritter in Zeiten begrenzter finanzieller Ressourcen neue Kriterien an jede Therapieform angelegt werden. Neue Kriterien, denen sich die manuelle Medizin stellen muss, aber auch erfolgreich stellen kann. Nur wenn die manuelle Medizin sich mit dem Maßstab der evidenzbasierten Medizin (EBM), wie sie u. a. vom Deutschen Cochran-Zentrum vertreten wird, messen lässt oder den Anforderungen z. B. des Instituts für Qualität und Wirtschaftlichkeit im Gesundheitswesen (IQWiG) genügt, können wir auf lange Sicht hoffen, dass die manuelle Medizin und damit Ihre tägliche Arbeit angemessen anerkannt und honoriert wird.

Um genau dies zu erreichen, hat die DGMM in den letzten Jahren zahlreiche Anstrengungen unternommen. Hierzu zählt zum einen die Einrichtung der Forschungsberatungsstelle in Jena, die der Stimulierung, Unterstützung und Beratung wissenschaftlicher Ansätze in der manuellen Medizin dient. Dies können Initi- ativen sowohl im universitären als auch im praktischen niedergelassenen Bereich sein. Hierzu zählt auch die Aufnahme der DGMM in die Arbeitsgemeinschaft der Wissenschaftlichen Medizinischen Fachgesellschaften e. V. (AWMF), um eine „Kommunikation mit anderen Fachgebieten auf Augenhöhe" sicherzustellen, wie Prof. Smolenski im Editorial zur Ausgabe 1/2009 der Zeitschrift Manuelle Medizin treffend festgestellt hat. Zusätzliche Studienprotokollpreise und finanzielle Forschungsförderungen können weitere Anreize schaffen, das wissenschaftliche Fundament der manuellen Medizin zu festigen und zu vertiefen.

\section{Studienergebnisse müssen nach EBM-Maßstäben belastbar sein}

Als Verlag sind wir uns der Tatsache bewusst, dass wissenschaftliches Arbeiten neben dem Klinik- oder Praxisalltag viel individuellen Enthusiasmus und große Opferbereitschaft der Forschenden verlangt. Dies gilt insbesondere dann, wenn die Ergebnisse der Arbeit letztlich auch den Ansprüchen an evidenzbasierte Medizin genügen und von Dritten als belastbar anerkannt werden sollen. Neben genau definierten Ein- und Ausschlusskriterien, im Vorfeld festgelegten Studienendpunkten und einer ausreichend großen Probandenzahl bedarf es einer großen methodischen Präzision, um am Ende mit den Ergebnissen das Fundament der manuellen Medizin nachhaltig zu festigen. Auch wenn viele von uns diese Umstände bedauern mögen und zu Recht die persönlichen Erfahrungen nicht mehr ausreichend gewürdigt sehen, wäre es im Sinne einer positiven Zukunft der manuellen
Medizin fahrlässig, diese Entwicklungen zu ignorieren.

Aus diesem Grund möchte Springer Medizin zukünftig durch die jährliche Verleihung des neu geschaffenen Julius-Springer-Forschungspreises Manuelle Medizin die Autoren besonders auszeichnen, die sich um das wissenschaftliche Fundament der manuellen Medizin besonders verdient gemacht haben. Verliehen wird dieser Preis an den Autor, der die auszeichnungswürdigste Originalarbeit zur Publikation in der Zeitschrift Manuelle Medizin eingereicht hat. Eine Jury, bestehend aus Prof. Dr. Smolenski, Präsident der DGMM, Dr. Matthias Psczolla, Präsident der DGMSM, und Dr. M. Frey, 1. Vorsitzender der MWE, bewertet dabei neben der wissenschaftlichen Qualität der Arbeit auch deren didaktische Umsetzung im Manuskript. Dotiert ist der Julius-Springer-Forschungspreis Manuelle Medizin mit 2000 Euro. Er wird erstmals im Rahmen des nächsten DGMM-Kongresses, 24.-26.09.2010 in Potsdam, verliehen werden.

Ich würde mich freuen, wenn dieser Preis einen kleinen Beitrag dazu leisten könnte, Forschung im Bereich der manuellen Medizin zu stimulieren und diese somit langfristig zum Wohle der Patienten als integralen Bestandteil der Schulmedizin zu bewahren.

Ihr

Jürgen Meyer zu Tittingdorf

Redaktionsleitung Manuelle Medizin

\section{Korrespondenzadresse}

Dr. J. Meyer zu Tittingdorf Springer Medizin Heidelberg Tiergartenstr. 17, 69121 Heidelberg juergen.meyerzutittingdorf@springer.com 